\title{
Authors' Response to the Letter to the Editor: Increased Circulating Angiopoietin-Like Protein 8 Levels Are Associated with Thoracic Aortic Dissection and Higher Inflammatory Conditions
}

\author{
Yunyun Yang ${ }^{1,2} \cdot$ Xiaolu Jiao ${ }^{1,2} \cdot$ Linyi $\mathrm{Li}^{1,2} \cdot$ Chaowei Hu${ }^{1,2} \cdot$ Xiaoping Zhang ${ }^{1,2} \cdot$ Lili Pan ${ }^{1} \cdot$ Huahui Yu ${ }^{1,2} \cdot$ Juan $^{\mathrm{Li}^{1,2}}$. \\ Dong Chen ${ }^{3} \cdot$ Jie $\mathrm{Du}^{1} \cdot$ Yanwen Qin ${ }^{1,2}$
}

Accepted: 04 May 2020 / Published online: 3 October 2020

(C) Springer Science+Business Media, LLC, part of Springer Nature 2020

\section{In Response}

We appreciate the letter submitted by Dr. Jia regarding our recently published article and very much appreciate the constructive suggestions. Dr. Jia raised an interesting point of whether this biomarker would be a predictor of risk for thoracic aortic dissection (TAD), that it may be used as a predictor of post-dissection complications. Dr. Jia also points out that it is unclear how area under the curve (AUC) calculations can be applied practically in a clinical setting; moreover, if the intent is to assess long-term risk prediction of TAD using Angiopoietin-like protein 8 (ANGPLT8), the current study design may be limited in addressing this question. Our study concluded that circulating full-length ANGPTL8 levels were an independent risk factor for TAD. ANGPTL8 combined with high-sensitivity C-reactive protein (hs-CRP) concentration and D-dimer may be a useful predictor of TAD, the cutoff value of ANGPTL8 was $>445.17 \mathrm{pg} / \mathrm{ml}$, the cutoff value of D-dimer was $>232 \mu \mathrm{g} / \mathrm{ml}$, and the cutoff value of hs-CRP was $>3.23 \mathrm{mmol} / \mathrm{l}$. As we mentioned in our discussion section, our research was a case-control study, we can only show

Yanwen Qin

qinyanwen@vip.126.com

1 Key Laboratory of Remodeling-related Cardiovascular Diseases, Beijing Institute of Heart, Lung and Blood Vessel Diseases, Beijing An Zhen Hospital, Capital Medical University, Beijing 100029, China

2 Key Laboratory of Upper Airway Dysfunction-related Cardiovascular Diseases, Beijing Institute of Heart, Lung and Blood Vessel Diseases, Beijing An Zhen Hospital, Capital Medical University, Beijing 100029, China

3 Department of Pathology, Beijing Anzhen Hospital, Capital Medical University, Beijing 100029, China associations not causality. The association of a combined higher ANGPTL8, D-dimer, and hs-CRP levels with a greater incidence of TAD needs to be verified in a larger sample size. We cannot assess long-term risk prediction of TAD using ANGPLT8; perhaps further prospective cohort studies can draw such conclusions.

Secondly, Dr. Jia suggested that we should consider investigating whether smoking may impact the association between ANGPTL8 and TAD. We strongly agree that smoking was association with inflammation and is a risk factor for aortic aneurysms/dissection. We analyzed the regression analysis by adding smoking risk factor, and the results were consistent with the finding in our original article. After adding smoking risk factor, increasing circulating ANGPTL8 levels was also associated with the occurrence $(\mathrm{OR}=1.569 / 100 \mathrm{pg}$ ANGPTL8, 95\% confidence interval $(\mathrm{CI})=1.102-2.232$, $P=0.012)$ and rupture risk $(\mathrm{OR}=2.461 / 100 \mathrm{pg}$ ANGPTL8, $95 \%$ confidence interval $(\mathrm{CI})=1.280-4.734, P=0.007)$ of TAD.

Lastly, Dr. Jia suggested that it is interesting if the authors can also assess the association between ANGPTL8 and thoracic aortic aneurysms without dissection. However, all patients in our research were undergoing conventional openheart surgery for TAD after pre-operative computed tomography angiography (CTA) diagnosis. CT results show aortic dissection formation, rather than patients with aneurysm expansion alone. So our patients in this study were TAD. We might collect thoracic aortic aneurysm to assess the association between ANGPTL8 and thoracic aortic aneurysms in the further study.

Publisher's Note Springer Nature remains neutral with regard to jurisdictional claims in published maps and institutional affiliations. 